\title{
La investigación sobre las conmemoraciones rituales en Colombia (siglos XIX-XXI): balance historiográfico
}

Sebastián Vargas Alvarez*

Resumen: El artículo presenta un estado de la cuestión de la producción investigativa contemporánea sobre las conmemoraciones rituales en Colombia desde el siglo XIX hasta el presente. Dada la estrecha relación que éstas conmemoraciones han mantenido con los proyectos de construcción y legitimación del Estado-nación durante los últimos dos siglos, algunos investigadores se refieren a ellos como "ceremonias cívicas" o "fiestas patrióticas". El texto se divide en tres partes. En un primer momento, se ubica el problema de las conmemoraciones rituales como un objeto de estudio que hace parte de una preocupación más general de la historia y las ciencias sociales por las políticas de la memoria y los usos públicos de la historia. Posteriormente, se presenta un inventario y análisis sucinto de los trabajos correspondientes a este objeto de estudio para el caso colombiano. La última sección reúne las conclusiones del balance, sugiriendo posibles nuevos caminos teóricos y metodológicos para la investigación de las conmemoraciones rituales.

Palabras clave: Conmemoraciones Rituales. Fiestas Patrióticas. Colombia. Historiografía.

\section{Delimitando un objeto de estudio}

La memoria colectiva se ha convertido en uno de los problemas más abordados en la historia y las ciencias sociales y humanas durante las últimas décadas. Su estudio sistemático fue propuesto

"Doutorando en Historia em la Universidad Iberoamericana Ciudad de México. E-mail: legionesdeclio@gmail.com

Anos 90, Porto Alegre, v. 22, n. 42, p. 207-235, dez. 2015 
por primera vez en los años veinte del siglo pasado por el sociólogo Maurice Halbwachs y por el historiador de la cultura Aby Warbug (ERLL, 2012). Sin embargo, el abordaje de la memoria colectiva sería abandonado y sólo retomado hasta los años setenta y ochenta, momento en el cual tiene lugar el fenómeno del "boom de la memoria". También conocido como "giro al pasado" o "giro subjetivo", el boom de la memoria obedece a la conjunción de dos factores, propios de la globalización capitalista y el auge de las industrias culturales: por un lado, la aceleración del tiempo y la experiencia histórica, la aparición de un nuevo "régimen de historicidad" presentista (HARTOG, 2007) a la sombra del cuál creció la preocupación por el patrimonio y se multiplicaron las conmemoraciones y los "lugares de la memoria" (NORA, 2008); y por otro, la irrupción del testimonio de la víctima/sobreviviente de grandes eventos traumáticos, principalmente la Shoa en Alemania, el Apartheid en Sudáfrica y las dictaduras militares en América Latina. Ambos fenómenos contribuyeron al agotamiento de la historiografía (nacional-oficial) como el único relato válido, autorizado y posible sobre el pasado (TRAVERSO, 2007).

Para los estudiosos de la memoria social, ha sido recurrente la pregunta por las políticas de la memoria y los usos públicos de la historia, que podría resumirse de la siguiente manera: “QQuién quiere que alguien recuerde y por qué? ¿A quién pertenece la versión del pasado que se registra y preserva?” (BURKE, 2011, p. 81). Dicha pregunta se circunscribe a los usos políticos del pasado que se despliegan en la esfera pública, especialmente en contextos marcados por eventos traumáticos, tales como la experiencia colonial, la guerra, el genocidio, la segregación, la desaparición forzada, etc.

Dentro de este campo de investigación existe un objeto de estudio relativamente menos explorado: el de las conmemoraciones rituales, en tanto uno de los medios o modalidades de transmisión de la memoria colectiva, cuya principal función es la reafirmación (o bien confrontación) de las identidades comunitarias mediante una acción performativa reiterada en el tiempo (CONNERTON, 2007). Se trata de fenómenos sociales en donde también se ponen en juego políticas de memoria y se utiliza y exhibe la historia de determinadas formas en el espacio público, y que evidencian 
tensiones y disensos en torno a la representación del pasado por parte de diversos sectores de la sociedad, especialmente en lo relativo a la definición de las identidades nacionales y/o colectivas (JELIN, 2002; GILLIS, 1994).

El presente trabajo presenta un estado de la cuestión de la producción investigativa contemporánea sobre los conmemoraciones rituales en Colombia desde el siglo XIX hasta el presente. Dada la estrecha relación que éstas conmemoraciones han mantenido con los proyectos de construcción y legitimación del Estado nación durante los últimos dos siglos, algunos investigadores se refieren a ellos como "ceremonias cívicas" o "fiestas patrióticas". La mayor parte de estudios revisados, de hecho, abordan los dos coyunturas conmemorativas más significativos en la historia del país: el Centenario y el Bicentenario de Independencia (1910 y 2010, respectivamente). El artículo se divide en tres partes. En esta breve introducción, se ubica el problema de las conmemoraciones rituales como un objeto de estudio que hace parte de una preocupación más general de la historia y las ciencias sociales y humanas por las políticas de la memoria y los usos públicos de la historia. Posteriormente, se presenta un inventario y análisis sucinto de los trabajos correspondientes a este objeto de estudio para el caso colombiano, a partir de una organización cronológica que contempla cinco momentos: las guerras de Independencia, el siglo XIX, el Centenario, el siglo $\mathrm{XX}$, y el Bicentenario. La última sección reúne las conclusiones del balance, sugiriendo posibles nuevos caminos teóricos y metodológicos para la investigación de las conmemoraciones rituales.

Este balance historiográfico tiene dos objetivos. Se presenta como una guía de estudio que pretende ser útil para los interesados en los problemas y objetos de investigación comentados más arriba, en donde se rastrean, exponen y analizan sucintamente las obras existentes sobre el tema. Así mismo, busca contribuir a un entendimiento más complejo y ampliado (en términos conceptuales y de delimitación espacio temporal) de las políticas de la memoria y los usos públicos de la historia, cuyos estudios generalmente se han limitado a los conflictos y traumas sociales de las últimas décadas del siglo XX (VARGAS, 2013). 


\section{Investigaciones sobre conmemoraciones rituales en Colombia}

\section{Los festejos fundacionales}

El origen de las fiestas patrias en Hispanoamérica se remonta al mismo período de las guerras de independencia (1808-1830), durante el cual son utilizadas como recursos simbólicos para la transmisión (y legitimación) de las nuevas ideas de nación, la creación de la identidad nacional y la representación y comunicación de una determinada imagen del poder y de la sociedad. Si bien su contenido cambia, su estructura es heredera del ceremonial cívico del período colonial (proclamaciones y juras, nacimientos y fallecimientos de miembros de la casa real, recibimiento de virreyes y gobernadores, etc.) (ORTEMBERG, 2013, p. 16).

Para este período, contamos con los trabajos de Marcos González Pérez. En Fiesta nacional y Guerra de Independencia neogranadina (2011), el autor explica cómo la fiesta cívica se convirtió en un escenario de lo político en plena guerra, utilizado tanto por los realistas como por los independentistas y describe algunas fiestas como el banquete ofrecido a Morillo en la capital (1816) o las festividades llevadas a cabo en Barichara y Tunja en honor a Bolívar luego de las victorias de Junín y Ayacucho (1825). En estos años de guerra son también importantes, en cuanto prácticas que se hacen públicas, la exhibición de trofeos conquistados al enemigo y la siembra de "árboles de la libertad" en el marco de congregaciones masivas. Finalmente, el texto da cuenta de la manera en que en el congreso de Cúcuta (1821) se decretó el 25 de diciembre como fiesta nacional oficial (en honor del "Salvador del mundo"), mucho antes de que el 20 de julio y el 7 de agosto (aniversarios de la proclamación de la independencia de 1810 y del triunfo de la batalla de Boyacá de 1819 , respectivamente) fueran oficializados como fiestas patrias en la década de los setenta del siglo XIX.

En Fiestas de independencia en Bogotá (1809-1830) (2014), por su parte, el autor analiza estos temas más de cerca para el caso de la capital. Allí plantea cómo no existe una gran ruptura, en cuanto a lo formal, entre las fiestas y carnestoladas tradicionales 
del período colonial y los festejos de la temprana república; no obstante las nociones de Libertad y Victoria aparecen como una novedad conceptual que marcará la especificidad de estos nuevos rituales, en donde se pasa de celebrar el retrato del rey para conmemorar al héroe patriota. El autor llega a la conclusión de que este es un momento de transición, en donde los símbolos y emblemas republicanos están aún en plena definición. Las honras fúnebres de Bolívar (1830), cierran este período "[...] durante el cual la relación guerra de independencia-fiesta nacional dejó de ser puesta en los escenarios imaginarios y, en su reemplazo, comenzó a difundirse una idea de República-Estado-Nación” (GONZÁLEZ, 2013, p. 168).

Otro estudio sobre este período es el de Georges Lomné sobre la influencia de la revolución francesa en la "simbólica del rito bolivariano" (1991). Según Lomné, el culto neogranadino al libertador y las ceremonias que lo rodean no sólo es el más acabado, duradero y extendido, sino también el más "contaminado" de los símbolos y conceptos políticos de la revolución francesa, si se lo compara con rituales republicanos similares en México y el Cono Sur. El gorro frigio, la figura de las ninfas y otras alegorías femeninas, los referentes a la mitología clásica, entre otros símbolos, pueblan el ritual republicano, y sólo adquieren sentido en el marco de una liturgia. Estos referentes coexisten y se hibridan con elementos del "tropismo americano", como la alegoría de la India-América o el mito solar de Bolívar, asociado a la cultura Inca.

En un interesante artículo sobre la (re)configuración de los conceptos de "Historia" y "Patria" dentro de la cultura política de Cartagena entre 1810 y 1814, Rafael Acevedo Puello (2010) explica cómo la institucionalización de "fiestas, paseos o bandos por las principales calles y plazas de la ciudad" se convirtió en una de las estrategias para "[...] evocar y presentar en público el nuevo Gobierno de la patria expresado en la recién decretada Constitución" (ACEVEDO PUELLO, 2010, p. 18). Así, refiere algunos desfiles y celebraciones cívicas que tuvieron lugar en la ciudad durante los años mencionados, entre otros usos públicos de la historia. 
La investigación sobre las conmemoraciones rituales en Colombia...

\section{El siglo XIX}

Sobre el desarrollo de las conmemoraciones rituales a lo largo del siglo XIX, existen algunos estudios. El texto de Santiago Díaz Piedrahita (2006), (ex presidente de la Academia Colombiana de Historia, recientemente fallecido), hace un recuento por la historia del establecimiento del 20 de julio como efeméride patria, desde 1820 , cuando comienza a celebrarse de manera no oficial, hasta su oficialización en la Ley 60 de 1873 . Se trata de un texto propio de la historiografía academicista tradicional, de tono apologéticonacionalista que, sin embargo, por su descripción de diversas conmemoraciones patrias (como la de 1849) y uso de diversas fuentes primarias - que no son cuestionadas - puede resultar útil para los investigadores de este campo. Otro trabajo interesante es el del brasilero Jaime de Almeida, quien dedica algunas páginas a la relación del culto a Santa Librada (santa patrona del día 20 de julio) y la fiesta de la independencia nacional en diferentes momentos del siglo XIX (DE ALMEIDA, 2010).

En La independencia como gesta heroica en el continuo histórico nacional: la 'densidad' de la representación, 1880-1909, Amada Carolina Pérez (2010) da cuenta de las fiestas cívicas de finales del siglo XIX y su particular representación de Bolívar. Su análisis de estas conmemoraciones es renovador en la medida en que hace énfasis en aspectos que no han sido aún trabajados con detenimiento en este tipo de estudios: la dimensión emotiva que producen las representaciones y prácticas propias del contexto conmemorativo; la condensación de diversas coyunturas históricas en una única conmemoración, lo cual contribuye a que el ritual se colme de una densidad de la representación1; las maneras en las que el pueblo es representado y el papel que se le otorga tanto en la gesta fundacional de la independencia como en el presente que la conmemora. La autora también desarrolla estas cuestiones a partir del análisis de otros soportes de la memoria oficial de la independencia que se constituyen en ese momento, como el Papel Periódico Ilustrado y la Galería de Notabilidades del Museo Nacional.

En su artículo "Padres de la Patria" and the ancestral past: commemorations of independence in nineteenth-century Spanish America, Rebecca Earle (1992) indaga sobre los usos del pasado

Anos 90, Porto Alegre, v. 22, n. 42, p. 207-235, dez. 2015 
ancestral (de las civilizaciones amerindias) en las conmemoraciones de la independencia durante el siglo XIX en Hispanoamérica (así como las tensiones entre esos usos y las representaciones de los sujetos indígenas del presente ${ }^{2}$ ), e incluye algunos episodios que corresponden al caso colombiano. Si bien en un primer momento (que corresponde con el de las guerras de independencia) hay una apropiación y exaltación activa del pasado ancestral, con el paso de las décadas la conmemoración de otros pasados (por ejemplo la Conquista) y de otros sujetos históricos (los padres de la patria: héroes de la independencia) cobrarán más importancia. Finalmente, la época del Centenario (1910) será la de la reconciliación con la "madre patria", que implica la indefinición o abierta negación del pasado indígena.

Para cerrar la sección correspondiente a este período, quisiera remitir a un reciente trabajo, Cultura política y conmemoración en Colombia: primer siglo de vida republicana, de Maite Yie y Amada Carolina Pérez (2012). Se trata de un texto que se concentra en el siglo XIX y la primera década del XX - hasta el Centenario -, y que no solamente aborda la conmemoración ritual sino también otras estrategias o procedimientos de administración estatal de la memoria (la escritura de la historia y la musealización de objetos). Las autoras ponen el énfasis en la relación entre conmemoración (actualización performativa del pasado) y formación de ciudadanía, y a partir de allí se describen y analizan conmemoraciones como la del 20 de julio de 1849, que contó con la activa participación de artesanos simpatizantes del gobierno liberal y fue escenario de la manumisión pública de esclavos; la del 20 de julio de 1873, año de su oficialización como fiesta patria oficial; la del natalicio de Simón Bolívar en 1881 y el IV Centenario del descubrimiento de América en 1892, marcadas ambas por el hispanismo y la reconciliación con el legado español-colonial; el Centenario de 1910 y el Centenario de la Batalla de Boyacá de 1919.

Este trabajo no sólo ofrece un panorama general de las políticas de la memoria y usos públicos de la historia en el proceso de formación del estado-nación en Colombia durante su primer siglo de vida republicana, sino que además propone una aguda reflexión sobre cómo estas políticas han mutado o permanecido 
hasta el presente, en donde las disputas por el sentido del pasado se complejizan en el contexto de la emergencia de "otras" memorias (también "purificadas" e incorporadas por el estado a la memoria nacional) en las últimas décadas: las de las minorías étnicas y las de víctimas/victimarios del conflicto armado interno. Por estas razones, considero que es una lectura introductoria, una entrada bastante recomendable para investigadores y estudiantes interesados por la problemática de las conmemoraciones.

\section{El Centenario}

La mayor parte de investigaciones sobre conmemoraciones rituales en Colombia corresponden al Centenario de Independencia (1910), patrón historiográfico compartido con otros países latinoamericanos, como México ${ }^{3}$. En general, todos estos estudios dan cuenta de la complicada coyuntura política que atraviesa Colombia en el período de cambio de siglo, tras la Guerra de los Mil Días y la pérdida de Panamá, lo que explica una política de "concordia" o "unidad nacional" y cierto sentimiento anti-norteamericano que se vieron reflejados en la preparación y ejecución de los programas conmemorativos (1907-1910). Así mismo, coinciden en señalar cómo el Centenario se presentó como una oportunidad a las elites para realizar un balance histórico-político del primer siglo de vida de la República, así como un espacio para exhibir y representar a la nación en el marco de una retórica de la modernización y el progreso que toma a la cultura europea como referente y ejemplo a seguir, y que adopta de manera especial el legado hispánico como fuente de cohesión social. Una buena síntesis puede encontrarse en el texto de Carolina Vanegas (2010), Representaciones de la Independencia y la construcción de una "imagen nacional" en la celebración del centenario en 1910.

Algunos estudios hacen énfasis en aspectos particulares de esta conmemoración. Por ejemplo, Fréderic Martínez (2000) en ¿Cómo representar a Colombia? De las exposiciones universales a la exposición del Centenario, 1851-1910, describe y analiza la Exposición Nacional, Industrial, Agrícola y Pecuaria, articulándola a una larga tradición de Ferias y Exposiciones Nacionales e Internacionales en 
las que participaba el país desde mediados del siglo XIX, con el fin de legitimar su entrada al "concierto" de las naciones "modernas" y "civilizadas". Así mismo, las diversas categorías de la exposición, su distribución en pabellones construidos en el recién inaugurado Parque de la Independencia (antes conocido como Bosque Reyes) y las narrativas modernizadoras y cosmopolitas que la atravesaron, son analizados por Luis Carlos Colón y Alejandro Garay en un libro editado por el Instituto Distrital de Patrimonio Cultural, La Ciudad de la Luz: Bogotá y la Exposición Agrícola e Industrial de 1910 (IDPC, 2005). ${ }^{4}$ Fabio Zambrano y Liliana Castelblanco (2002), a su vez, hacen la historia de la única huella material del Centenario que pervivió en este parque durante el siglo XX y hasta la actualidad: el kiosco de la luz, uno de los primeros edificios construidos con cemento en la ciudad y, junto con los primeros tendidos de luz eléctrica, símbolo de la "modernidad" que arribaba en 1910.

Juan Camilo Escobar, a su vez, analiza el papel que desempeñaron el arte y los artistas en la Centenario, tanto en la exposición (para la cual se destinó un pabellón de Bellas Artes en donde fueron exhibidas obras de noventa y nueve artistas), como acompañando desde lo estético y creativo las diversas actividades conmemorativas: ceremonias religiosas y muestras de industria y agricultura, óperas, corona solemne para la estatua de Bolívar, y procesiones. El afamado pintor Andrés de Santamaría (por entonces director de la Escuela Nacional de Bellas Artes) fue nombrado por la Comisión Nacional del Centenario para coordinar la parte artística de la festividad (ESCOBAR, 2011, p. 304-305). Es interesante señalar que la exposición artística, junto con los gestos de beneficencia para con los menos afortunados, fueron de los pocos espacios de la conmemoración en los que las mujeres tuvieron un rol protagónico, pues del total de artistas participantes, 36 eran mujeres. Escobar también se detiene en la importancia que tuvieron la literatura y los concursos literarios durante el centenario, y de su texto se concluye que tanto el arte como la literatura desplegaron los ideales de cultura, progreso, belleza, y verdad con los que se quería asociar a la nación colombiana.

Sobre la exposición, finalmente, debe mencionarse el interesante proyecto interdisciplinar de Jimena Montaña y Pablo Castillo 
(2010), el corto-documental El fantasma de la libertad: Celebraciones por la Independencia, ganador de una convocatoria del IDPC. Ha partir de la investigación histórica, el empleo de fuentes primarias visuales tales como planos y fotografías, y la animación en $2 \mathrm{D}$ y $3 \mathrm{D}$, se reconstruye el contexto del Centenario, y de manera especial, la apariencia (interior y exterior) de los diversos pabellones, kioscos y espacios construidos y dispuestos en el Parque Nacional. Se trata de un documento central no sólo para la investigación, sino también para la docencia y difusión, por su capacidad de estimulación de la imaginación y la sensibilidad históricas, además de su asequibilidad, pues es de libre acceso a través del portal YouTube.

En otro libro, Carolina Vanegas explora hábilmente algunas disputas simbólicas entre diversos sectores sociales con respecto a la conmemoración de la independencia en 1910, y para ello hace una historia de la escultura conmemorativa, tomando dos casos de estudio: la estatua ecuestre de Simón Bolívar emplazada en el parque de la independencia (lugar en que, como puede verse, convergieron múltiples lugares de memoria en el Centenario) y la estatua a Policarpa Salavarrieta, instalada en el barrio Las Aguas. La autora identifica una tensión entre ambos proyectos: mientras que la estatua de Bolívar representa una propuesta oficial, estéticamente eurocentrada (se contrata para realizarla al francés Emanuel Frémiet) y espacialmente central, la de la Pola es una iniciativa popular, ubicada en un lugar periférico de la ciudad y obra de un escultor colombiano, Dionisio Cortés.

El énfasis en las disputas simbólicas entre diversos sectores de la sociedad a partir de las esculturas conmemorativas de Frémiet y Cortés permite relativizar la unidad de los discursos hegemónicos, así como evidenciar el papel de otros actores en dicha celebración. Desde ese punto se reconstituyó en la presente investigación un entramado de tensiones entre artistas y comitentes, con la intención de mostrar cómo se pusieron en juego no sólo los intereses políticos e ideológicos, sino las ideas estéticas referidas a la escultura y a la construcción de la imagen de la ciudad vigentes durante la primera década del siglo XX en Colombia (VANEGAS, 2012, p. 122). 
Otra vertiente de estudios sobre el Centenario, se enfoca en las dimensiones regionales de dicha conmemoración. Algunos, se concentran en las disputas sostenidas entre las elites bogotanas y las elites de algunas regiones sobre el sentido del festejo, e incluso sobre la definición la oficialidad de las fechas a conmemorar, como los trabajos de Raúl Román (2010) y Rafael Acevedo Puello (2011) para el caso de Cartagena. Otros, dan cuenta de las agendas conmemorativas en contextos provinciales, como el artículo de Abel Martínez sobre las conmemoraciones centenarias de 1910 y 1911 en Tunja (2012), o el de Álvaro Acevedo Tarazona, que remite someramente a las características de los festejos en Bucaramanga (2010). Por último, Fiestas, memoria y libros: las ediciones conmemorativas del primer centenario de la Independencia en Bogotá y Cali, de Juan David Murillo Sandoval (2012), presenta un análisis comparado de las memorias oficiales de los festejos en estas dos ciudades, mostrando las particularidades de la auto-representación de cada una de ellas en sus crónicas centenarias.

Cachacos y guaches: la plebe en los festejos bogotanos del 20 de julio de 1910, de Alexander Pereira (2011), se pregunta por la participación de los sectores populares de la capital en la conmemoración, y por la actitud de las elites capitalinas frente a estas "clases marginadas", concluyendo que el pueblo fue excluido sistemáticamente de las diversas actividades de la agenda conmemorativa, y su papel se limitó al de mero objeto de beneficencia por parte de los organizadores del festejo y las clases acomodadas de la ciudad (especialmente, las mujeres, "damas de sociedad"). Ante la ausencia de diversiones populares en la agenda oficial, se llevaron a cabo fiestas de manera espontánea en barrios periféricos como Las Cruces, pero éstas, recuerda Pereira (2011), no están lo suficientemente documentadas.

Por último, podemos citar un reciente volumen colectivo sobre los Centenarios en Latinoamérica, editado por Stephan Scheuzger y Sven Schuster (2013), que incluye una introducción sobre el estudio historiográfico de las conmemoraciones centenarias a cargo Scheuzger; un análisis sobre el papel privilegiado del hispanismo y la lengua castellana en la efeméride colombiana, escrito por Andrés Jiménez; y un capítulo sobre los centenarios en Bogotá y Caracas, de Thomas Fisher. 
La investigación sobre las conmemoraciones rituales en Colombia...

\section{El siglo XX}

El período del siglo XX, comprendido entre la celebración del Centenario (1910) y la del Bicentenario (2010), es el menos estudiado en lo que respecta a nuestro objeto de investigación. Quizás el texto más completo que se ocupa de esta época es el de la antropóloga Maite Yie (2010), Narrativas de pasado, de nación y ciudadanía en las celebraciones patrióticas durante el siglo XX en Colombia. ${ }^{5} \mathrm{Su}$ estudio comienza recordando la historicidad de toda conmemoración (esto es, cómo éstos están atravesados por las disputas simbólicas entre actores sociales que en el presente contienden por la definición del pasado, a partir de sus intereses y agendas políticas en el presente), una precaución teórica que ningún estudioso sobre este tema debería pasar por alto:

[en las conmemoraciones] se escenifican, confrontan y negocian diversas narrativas sobre el proceso en el que se habría gestado la patria, reivindicadas por distintos sectores sociales, hegemónicos y subalternos, en los ámbitos nacional, regional y local [...] en las obras, eventos y discursos realizados como parte de dichas celebraciones circulan representaciones sobre los personajes, las escenas y los escenarios que están ligados a dicho proceso y sobre su participación y articulación dentro del mismo [...] en las fiestas patrióticas se movilizan diversas narrativas sobre el presente, siendo la escenificación del pasado sólo uno de los recursos para proponer diversos guiones sobre la nación y las jerarquías de interacciones entre sus ciudadanos (YIE, 2010, p. 131).

Desde esta perspectiva analítica, la autora examina las siguientes conmemoraciones: fiestas del período del Centenario (1910-1922) en ámbitos locales (1911 en Cartagena, 1919 en Boyacá y 1922 en Bomboná, Nariño); el Centenario de la muerte de Bolívar bajo el gobierno de Olaya Herrera (1930), y el Sesquicentenario de la Independencia bajo el Frente Nacional (1960). Su estudio llama la atención sobre actores e instituciones sociales hegemónicas que intentaron detentar el monopolio sobre la significación de los festejos

Anos 90, Porto Alegre, v. 22, n. 42, p. 207-235, dez. 2015 
patrios a lo largo del siglo: la Academia Colombiana de Historia, la escuela, la Iglesia; también sobre cómo la ceremonia patriótica del siglo XX se caracterizó por la interacción de tres rituales: el cívico, el religioso y el castrense.

En el artículo El centenario de la muerte del "hombre de las leyes": el papel del Centro de Historia de Santander y la conmemoración departamental (1940), Gabriel Samacá (2013) se aproxima a los actos conmemorativos en honor a Francisco de Paula Santander que tuvieron lugar en Bucaramanga y otras localidades del departamento santandereano entre 1939 y 1940. Samacá destaca el cariz pedagógico de dicha conmemoración, expresado en el papel privilegiado que tuvieron las escuelas y los proyectos editoriales de los académicos del Centro de Historia de Santander en los eventos del Centenario.

El trabajo de Carlos Rincón (2012), Conmemoraciones y crisis: Centenario, Sesquicentenario, Bicentenario ofrece un análisis de cómo el período de la independencia es reconsiderado en tres momentos de crisis: 1910, 1960 y 2010. En el Sesquicentenario, Rincón evidencia una tensión entre la reproducción de la historia patria en tanto narrativa mítica de lo nacional - expresada en la inauguración del Monumento a los héroes y del Museo del 20 de julio en Bogotá - y la aparición de un cierto revisionismo histórico, presente en la pluma de autores como Indalecio Liévano. Así mismo, explica que las imágenes, que circularon a través de todas estas conmemoraciones, se convirtieron en parte fundamental de la "memoria cultural" de los colombianos. Su texto finaliza con una sugerente reflexión sobre la plaza pública como escenario por excelencia de la conmemoración ritual y la contienda política.

Alexander Betancourt también aborda el tema del revisionismo histórico durante el Sesquicentenario (2011), en Pasado nacional y revisionismo histórico: lecturas sobre la independencia en los años sesenta en Colombia y México. Luego de introducir al lector en la relación entre escritura de la historia y conmemoración (relación que es una de las expresiones de los usos públicos de la historia, o de la dimensión pública del discurso histórico), el autor da cuenta de la década de los sesenta como un momento conflictivo, en donde se combinaron la legitimación del compromiso intelectual y la militancia política (estimulada, entre otros fenómenos, por 
el triunfo de la revolución cubana y su influencia en las nuevas generaciones); la profesionalización de la disciplina histórica; y el afán del Frente Nacional por presentar una lectura de la historia armoniosa que rescatara los "verdaderos valores nacionales", a partir de la orientación de la Academia Colombiana de Historia y las Juntas de los Festejos de 1960. Estos elementos en tensión permiten entender cómo "[...] la escritura de la historia participó como una herramienta a favor del monumento y la conmemoración pero, al mismo tiempo, también fue un instrumento de la polémica como una fuente de interpretación crítica del pasado y el presente nacional” (BETANCOURT, 2011, p. 122).

\section{El Bicentenario}

Sobre los festejos del Bicentenario de Independencia, como podría esperarse por su proximidad en el tiempo, las investigaciones aún son escasas. Con ocasión del bicentenario se desató un verdadero boom editorial, una reactivación de la historiografía sobre el período de la independencia, en medio de la cual aparecieron numerosas investigaciones, reediciones de trabajos anteriores, $\mathrm{y}$ reimpresiones de documentos de o sobre dicha época. Muchas de estas publicaciones se caracterizaron por ser críticas, por abordar el pasado independentista desde nuevos ángulos e incluyendo actores, procesos y problemas antes ignorados. ${ }^{6}$ Algunos de estos textos, en sus prólogos e introducciones, aluden al bicentenario - y a las coyunturas conmemorativas en general - como momentos de revisión de la historia y la cultura y de reflexión política intensa, oportunidad que no puede ser desaprovechada al darle prioridad al otro componente de las conmemoración, lo festivo, que puede convertirse en espectáculo banal (PALACIOS, 2009; CALDERÓN; RESTREPO, 2010). No obstante, y sin demeritar el valor de estas interesantes consideraciones, es importante señalar que se trata de textos sobre el proceso histórico de la independencia, y no sobre el bicentenario, por lo que sólo sirven indirectamente al interesado en las conmemoraciones rituales.

Reflexiones similares, sumadas a otras que ponen el acento en la exclusión de los sectores subalternos de los relatos y de los imaginarios de la sociedad colombiana, pueden encontrarse en el 
artículo ¿Quién imagina la independencia? A propósito de la celebración del bicentenario en Colombia, de Eduardo Restrepo (2010). Allí se abordan tres aspectos: primero, la vigencia de las estructuras de la colonialidad en Colombia, que no se trascienden en el siglo XIX (con la independencia) y se mantienen incluso hasta el día de hoy; segundo, la "necesidad de deselitización de las genealogías de la colombianidad, incluyendo lo que aparece como Independencia"; y tercero, la comprensión de los eventos conmemorativos a partir de las preocupaciones e historicidad de quienes celebran (de su presente) (RESTREPO, 2010, p. 69).

Concretamente sobre las políticas de la memoria y los usos públicos de la historia desplegados durante la conmemoración bicentenaria, puede citarse el artículo de Sebastián Vargas (2011), El Bicentenario de la Independencia en Colombia: rituales, documentos, reflexiones. El texto remite al contexto de creación de la Alta Consejería para el Bicentenario de Independencia (entidad encargada de diseñar, coordinar y ejecutar los festejos), desde 2008; y da cuenta de las principales actividades conmemorativas oficiales, así como de las conmemoraciones alternativas protagonizadas por sectores disidentes y movimientos sociales, como la Marcha Patriótica, el Congreso de los Pueblos y organizaciones afrodescendientes, entre 2009 y 2010. Este es el primer trabajo en abordar los principales usos y representaciones de la historia que circularon en la esfera pública durante el bicentenario, y de manera particular, las disputas $\mathrm{y}$ tensiones entre diversos actores sociales en torno a la definición del pasado y su apropiación en el presente.

En otro trabajo, Cabalgando entre el pasado y el presente. Usos estatales del bicentenario en Colombia (2009-2010), Vargas toma como caso de estudio las propuestas conmemorativas del ejército nacional ("Ruta libertadora” y "200 años de honor y gloria"). El argumento central del texto es que el bicentenario fue utilizado, por parte de los publicistas del Ministerio de Defensa y la Presidencia de la República, como un instrumento de legitimación del modelo de seguridad democrática del gobierno de Álvaro Uribe Vélez (20022010), por la vía de la exaltación del patriotismo y la fabricación de una línea de continuidad entre los ejércitos patriotas y el ejército nacional contemporáneo (VARGAS, en prensa). 
Por su parte, el reconocido historiador Jorge Orlando Melo, publicó en su página web un breve documento titulado La celebración de la independencia: espectáculo y reflexión. Allí, Melo (2011) compara el contexto actual colombiano con el de 1910 (año de conmemoración del centenario), y refiere someramente a algunas de las actividades y conmemoraciones que tuvieron lugar en el 2010, como las exposiciones del Museo Nacional, la Biblioteca Nacional y la Biblioteca Luis Ángel Arango, así como del proyecto educativo Historia Hoy, del Ministerio de Educación Nacional. Pero ante todo, se detiene en la producción historiográfica reciente sobre el período de la independencia.

Finalmente, la revista de antropología de la Universidad Nacional, Maguaré, incluyó en su número 24 una sección sobre la conmemoración de la independencia desde la perspectiva de sectores subalternos, con una introducción de los investigadores Marta Saade y Pablo Mora (2010). Se trata de textos de líderes indígenas y afrodescendientes como Darío Mejía Montalvo, Feliciano Valencia, Juan de Dios Mosquera, Jeremías Tunubalá y Liliana Pechené, Lorenzo Muelas y Carlos Rosero. Observaremos dos ejemplos. En No estamos para celebraciones: las lecciones aprendidas de los 200 años, el líder nasa Feliciano Valencia (2010) expone cómo durante los últimos doscientos años los pueblos indígenas han sido objeto de una "victimización sistemática", a la vez que han llevado a cabo constantes "ejercicios de resistencia" para subsistir como comunidad. Razones suficientes para mantenerse al margen de la celebración bicentenaria, y en vez de eso, utilizar la coyuntura para proponer un "nuevo país" de la mano de otros sectores sociales (VALENCIA, 2010, p. 409). Una perspectiva similar se encuentra en el artículo de Darío Mejía Montalvo, de la comunidad zenú, quien además denuncia el multiculturalismo que caracterizó al programa oficial de festejos, al denominarlo "disfraz de la diversidad” (MEjÍA, 2010). Estos documentos son ejemplos - entre muchos otros - que dan cuenta de las tensiones, contradicciones y disputas sobre el sentido de lo conmemorado en 2010. 


\section{Posibles caminos: cuestiones teóricas, precauciones metodológicas}

Las investigaciones hasta aquí reseñadas son bastante heterogéneas si se miran en conjunto: abordan diferentes momentos a lo largo de un lapso amplio de tiempo - los últimos dos siglos -; abarcan diversas locaciones geográficas del territorio nacional; sus enfoques teóricos y analíticos oscilan entre una historia política clásica, la historia cultural y la antropología histórica; y emplean diversas fuentes para reconstruir las conmemoraciones. Más allá de sus diferencias o especificidades, todas nos ofrecen relevantes aproximaciones, descripciones y análisis de las ceremonias patrióticas o cívicas, insumos pertinentes para una historia y una crítica de la conmemoración ritual en Colombia. Para cerrar este balance, quisiera plantear algunas cuestiones teóricas y precauciones metodológicas, a modo de problemas interconectados, en aras de una comprensión más crítica y compleja de la investigación hecha hasta el momento, así como de la apertura de nuevos senderos de indagación.

El primer problema es el del desequilibrio en términos de los períodos abordados. Como ya se señaló antes, y como queda patente en este estado del arte, la mayoría de investigadores se han concentrado en el Centenario de independencia (1910), dedicando menor atención al siglo XIX, y en especial, al siglo XX. El período de comienzos del siglo XIX, correspondiente a las primeras fiestas y rituales republicanos, está mejor cubierto dado el interés reciente en la historiografía colombiana por la emergencia de una nueva cultura política durante la Independencia, la cual fue estructurada por discursos y prácticas que circularon en el ceremonial patriótico, entro otros medios y objetos culturales (ORTEGA; CHICANGANÁ, 2011, 2012). Quedan pendientes, por tanto, el emprendimiento de nuevas investigaciones sobre conmemoraciones rituales en el siglo $\mathrm{XX}$, en especial durante la segunda mitad del mismo (particularmente de los sesenta a los noventa), momento en que las tendencias de la globalización y el multiculturalismo comienzan a transformar lentamente las bases de la nacionalidad que habían dado sustento a la fiesta patriótica desde sus comienzos, permitiendo la emergencia de nuevas ciudadanías y diversos discursos sobre el pasado 
La investigación sobre las conmemoraciones rituales en Colombia...

nacional conmemorado (YIE, 2010, p. 133). Estas transformaciones y disputas en torno al paradigma de nación (y del significado de la ciudadanía), así como sus implicaciones en los contenidos y las formas de las conmemoraciones, se manifestaron claramente durante el bicentenario de independencia. 2010, en consecuencia, es otro momento que espera por ser estudiado sistemáticamente por futuros investigadores.

De la misma manera como deben explorarse otros puntos en el tiempo además del Centenario, resulta importante descentrar a Bogotá como sede por antonomasia de la conmemoración ritual. Por supuesto que la capital de la república fue el escenario de los principales eventos conmemorativos y sede de las instituciones y legislaciones que los regularon por dos siglos. No obstante, desconocer qué sucedió en otras ciudades y provincias es ignorar la agencia histórica y política de las regiones, sus propias agendas conmemorativas y concepciones y usos particulares de la historia. En este sentido, son rescatables los estudios sobre Tunja, Santander, Cartagena y Cali mencionados más arriba, pero hacen falta nuevos trabajos para otras regiones que nos permitan conocer mejor nuestra historia de conmemoraciones. Adicionalmente, éstos deben intentar acercarse a las conmemoraciones en toda su complejidad, sin limitarse a reconstruir la experiencia de las elites regionales (y su posición frente a los lineamientos conmemorativos provenientes del centro) para dar cabida a otros sujetos sociales, especialmente a los sectores populares y subalternos.

Esto nos lleva a nuestro tercer problema: las fuentes. Generalmente, las fuentes disponibles para nuestro objeto de estudio, son producidas por las elites y dan testimonio del papel de las mismas en la organización y ejecución de los festejos, así como su idea de la historia. Rara vez dan cuenta de la participación de otros actores sociales, especialmente en las conmemoraciones del siglo XIX y gran parte del XX. Para las últimas décadas del siglo XX y, en particular para el Bicentenario, encontramos una mayor cantidad y diversidad de fuentes, así como un reconocimiento mayor de la participación de múltiples sectores participantes, debido al cambio de paradigma de nación y al "giro multicultural" que caracteriza a los festejos de este período.

Anos 90, Porto Alegre, v. 22, n. 42, p. 207-235, dez. 2015 
Fundamentalmente, han sido dos los tipos de fuentes las utilizadas en estas investigaciones: por un lado, las crónicas oficiales, producidas por las Juntas y/o Comisiones para los festejos, así como por los gobiernos central y/o departamentales y municipales; ${ }^{7}$ $y$, por otro, la prensa, tanto nacional como local. Ambos tipos de fuentes pueden encontrarse en bibliotecas, archivos, fuentes de documentación y hemerotecas. Para rastrar los documentos de prensa y otras fuentes primarias, es imprescindible tener en cuenta las fechas conmemorativas, concentradas en los meses de julio y agosto. Adicionalmente, cada región o ciudad cuenta con su propia efeméride (el 11 de noviembre para Cartagena, 7 de abril para Bomboná, etc.). Por otra parte, algunos de los estudios reseñados utilizan fuentes visuales: exploran las conmemoraciones a partir de su registro iconográfico y/o fotográfico (PÉREZ, 2010; LOMNÉ, 1991). Finalmente, los que abordan conmemoraciones más recientes, se valen de la observación etnográfica (VARGAS, 2011).

Considero pertinente seguir teniendo en cuenta las fuentes tradicionales, interrogándolas con nuevas preguntas para poder escuchar otras voces sobre las conmemoraciones. En los intersticios de las crónicas y memorias oficiales, así como de los reportajes de prensa, es posible hallar otros puntos de vista e información inédita sobre las celebraciones. Así mismo, es recomendable abrirse a nuevos tipos de documentos: audiovisuales (por ejemplo archivos de transmisiones de televisión), multimedia (como las páginas web oficiales montadas para el bicentenario), registros etnográficos y la memoria colectiva: entrevistas y discusiones focales con grupos de diferentes edades que permitan rastrear el recuerdo (y los olvidos) de la experiencia conmemorativa (estos últimos para el caso de las fiestas cívicas de la segunda mitad del siglo XX y del XXI), etc.

Quizás el problema central, y más complicado de resolver, es el de la necesidad del descentramiento de la nación como sujeto de la historia de las conmemoraciones rituales. ¿Cómo hacer la historia de otras conmemoraciones, disidentes, a otras escalas, de otras cosas, cuando lo que se ha documentado durante las dos últimos siglos y lo que se ha investigado en las tres últimas décadas han sido casi exclusivamente las fiestas patrióticas? Me parece que un referente conceptual que puede contribuir a pensar otro tipo 
La investigación sobre las conmemoraciones rituales en Colombia...

de discursos y prácticas conmemorativas es el trabajo de François Hartog (2007), quien propone la noción de "regímenes de historicidad". Este concepto alude a las diversas maneras en las que una sociedad se relaciona con el tiempo, es decir, abre la posibilidad de indagar otras formas de experiencia y otras temporalidades no necesariamente atadas al tiempo vacío y homogéneo de la nación moderna (aquel que ha dictado el ritmo de las conmemoraciones rituales desde el siglo XIX). Desde esta perspectiva, trabajos clásicos como el de Joanne Rapapport (2000), La Política de la memoria: Interpretación indígena de la historia en los Andes colombianos, y más recientes como el de Pablo Gómez sobre la fiesta del Zocán en Sogamoso (2011), caben en este balance historiográfico y sirven de inspiración para el estudio riguroso y sistemático de conmemoraciones ajenas o incluso contrapuestas a las lógicas conmemorativas del estado-nación. Se trata de enfoques y estudios de caso que han sido desarrollados principalmente desde la antropología, de la cual los historiadores podemos aprender un método y un tratamiento de fuentes a las que no estamos suficientemente acostumbrados, con el fin de comprender y explicar otras texturas y otros sujetos de la conmemoración. Un texto clave para propiciar este acercamiento sigue siendo la conferencia que E. P. Thompson (2000) diera en la India hace casi 40 años, Historia y antropología.

Es importante señalar que una de las modalidades de conmemoración no necesariamente asociada al ritual patriótico más común en las últimas décadas es la relacionada con los procesos de recuerdo/olvido colectivo y elaboración del duelo en contextos postraumáticos. Movimientos sociales, organizaciones de Derechos Humanos y otros actores sociales han irrumpido en la esfera pública para realizar actos de rememoración crítica de eventos límite (genocidio, desaparición forzada, masacres, etc.) y exigencias de justicia, reparación y verdad, a partir de propuestas estéticas y/o performativas con un claro contenido histórico combinadas con formas más tradicionales de protesta política (JELIN; LONGONI, 2003). Estas conmemoraciones alternativas, o "golpes de memoria" (EL COLECTIVO, 2009), constituyen una nueva veta de investigación que permite dar cuenta de las disputas simbólicas sobre el sentido del pasado. Así, por ejemplo, podemos citar el libro de Katherine Hite (2012), Politics and the art of commemoration: Memorials to

Anos 90, Porto Alegre, v. 22, n. 42, p. 207-235, dez. 2015 
struggle in Latin America and Spain, en donde se estudian este tipo de prácticas y discursos (contra)conmemorativos para el caso de Argentina, Chile, Perú y España. En Colombia existe una amplia literatura sobre el conflicto armado interno, las víctimas de la violencia, y los procesos de construcción de la memoria colectiva (e, inclusive, el papel del arte en estos procesos). Sin embargo hacen falta estudios que aborden específicamente el problema de las fechas, la temporalidad y la conmemoración.

Por otra parte, habría que tener en cuenta que esta modalidad de conmemoración ritual, cuyo sujeto ya no es el estado-nación sino la "víctima", es en muchos casos incorporada a la memoria nacional a través de políticas de memoria oficiales. Por ejemplo, el Artículo 142 de la Ley de Víctimas y Restitución de Tierras proclamó recientemente el 9 de abril (fecha que evoca el magnicidio de Jorge Eliécer Gaitán en 1948) como "Día nacional de la memoria y la solidaridad con las víctimas” (COLOMBIA, 2011, p. 76). Por lo tanto, los investigadores de este tipo de conmemoración deben estar bastante atentos a los conflictos, negociaciones y desplazamientos en torno a las efemérides y su significado histórico, que tienen como protagonistas a los diversos sectores sociales que promueven "golpes de memoria” en el espacio público y a las instituciones gubernamentales que tratan de administrar estas nuevas manifestaciones de la memoria colectiva.

Finalmente, faltaría señalar un último desplazamiento conceptual necesario para abrir nuevos rumbos investigativos sobre las conmemoraciones rituales: pasar del estudio de la producción de los discursos y las prácticas conmemorativas al estudio de su recepción. La mayoría de estudios referidos en este balance, se aproximan a las fiestas nacionales o cívicas como formas culturales dominantes producidas por las elites, en donde las categorías de "nacionalismo", "progreso" o "ciudadanía" jugaron un papel determinante. Un lenguaje conmemorativo aparentemente hegemónico y homogéneo desplegado en diversos soportes - poesía, monumentaria, museografía, historiografía, etc. - del que ha quedado testimonio, principalmente, en crónicas oficiales y reportajes periodísticos. No obstante, sabemos menos de la forma en que los sectores sociales no pertenecientes a las elites se apropiaron, usaron, interpretaron (o no) estos discursos, gestos y prácticas conmemorativos que el 
La investigación sobre las conmemoraciones rituales en Colombia...

estado-nación confeccionó en diferentes momentos de su historia para el consumo y formación de sus ciudadanos, básicamente porque por lo menos hasta la segunda mitad del siglo XX estuvieron excluidos tanto de los festejos como de su registro (PEREIRA, 2011).

Se trata de un problema historiográfico bastante conocido en - la historia cultural, y que ha sido planteado por Roger Chartier, en un libro donde define la cultura como apropiación, abriendo la posibilidad de rastrear las dimensiones de resistencia y resignificación de toda forma cultural "dominante":

[...] pensar las prácticas culturales en términos de apropiaciones diferenciadas autoriza también a no considerar como totalmente eficaces y radicalmente aculturantes los textos, las palabras o lo ejemplos que se proponen moldear los pensamientos y las conductas de la mayoría. Esas prácticas son siempre creadoras de usos o de representaciones en modo alguno reductibles a las voluntades de los productores de discursos y de normas. De ninguna manera el acto de lectura puede por tanto ser anulado en el texto mismo, ni los comportamientos vividos en las prohibiciones y los preceptos que pretenden regularlos. La aceptación de los modelos y de los mensajes propuestos se operan a través de adecuaciones, rodeos y en ocasiones resistencias que manifiestan la singularidad de cada apropiación (CHARTIER, 1995, p. 12).

Esta conceptualización de la cultura como apropiación nos permite entonces entender la conmemoración como un fenómeno heterogéneo y complejo, un texto que no es incontestado, un gesto que no siempre es seguido al pie de la letra. Metodológicamente, este cambio de perspectiva implica buscar en los silencios de las fuentes tradicionales así como en la riqueza de los nuevos registros documentales, aquellas voces, pistas e indicios que contribuyan a reconstruir los diversos usos, reapropiaciones y representaciones de la conmemoración que escaparon a la intención y al sentido originario que le asignaron sus productores. Toda futura indagación sobre conmemoraciones rituales debería considerar estos desplazamientos y rearticulaciones conceptuales y metodológicas.

Anos 90, Porto Alegre, v. 22, n. 42, p. 207-235, dez. 2015 


\section{RESEARCH ON RITUAL COMMEMORATIONS IN COLOMBIA (XIX-XXI CENTURIES): A HISTORIOGRAPHICAL BALANCE}

Abstract: This paper presents a historiographical balance of contemporary research on commemorative rituals in Colombia since the nineteenth century to the present. Given the close relationship these rituals have had with nation -state building and legitimation over the past two centuries, some researchers refer to them as "civil ceremonies" or "patriotic holidays". The article is divided into three parts. At first, the problem of ritual commemorations is defined as an object of study that is part of a more general concern of history and social sciences about the politics of memory and public uses of history. Later, it is presented an inventory and brief analysis of contemporary inquiries on this subject in Colombia. The final section brings together the conclusions of the balance, suggesting possible new theoretical and methodological paths on ritual commemorations research.

Keywords: Ritual Commemorations. Patriotic Holidays. Colombia. Historiography.

\section{Notas}

1 "Resulta interesante además que en la fiesta que conmemora un acontecimiento en particular se condensen diferentes coyunturas del proceso independentista: el régimen del terror y la campaña militar, por ejemplo. La celebración quiebra así la cronología y abre una lógica temporal diferente en tanto aglutina momentos diversos en un mismo espacio simbólico" (PÉREZ, 2010, p. 81).

2 "Al incorporar el pasado indígena en la narrativa histórica nacional, se valoró la civilización precolombina, mientras que al mismo tiempo se separó a los indígenas contemporáneos de ese legado de la pre-conquista, el cual no podían entender ni apreciar porque estaban oprimidos” (EARLE, 2002, p. 792). La traducción es mía. ${ }^{3}$ En el marco de mi investigación doctoral, he realizado para el caso mexicano un rastreo similar al presentado en este balance. De los 31 estudios que he podido identificar y consultar, 12 corresponden a los Centenarios de 1910 y 1921. ${ }^{4}$ Luis Carlos Colón (2012) desarrolla las mismas temáticas en otro lugar: Representar la nación en el espacio urbano: Bogotá y los festejos del Centenario de la Independencia.

${ }^{5}$ Es importante señalar que este texto, al igual que los antes reseñados de Amada Carolina Pérez (2010) y Carolina Vanegas (2010), forman parte de la investigación curatorial de la exposición Las historias de un grito. Doscientos años de ser colombianos (presentada por el Museo Nacional de Colombia con motivo del Bicentenario de Independencia), y se encuentran publicados en el catálogo de la misma.

Anos 90, Porto Alegre, v. 22, n. 42, p. 207-235, dez. 2015 
La investigación sobre las conmemoraciones rituales en Colombia...

${ }^{6}$ Para un acercamiento a este corpus historiográfico, pueden verse los estados del arte de Germán Mejía (2011) y Jorge Orlando Melo (2011).

${ }^{7}$ Las instituciones encargadas de las conmemoraciones oficiales han dejado a su paso unos anales que permiten rastrear las políticas de la memoria en momentos específicos de la historia del país. Por ejemplo, la Academia Colombiana de Historia ha publicado discursos y programas conmemorativos para diferentes años del siglo XX. Ver, por ejemplo, ACADEMIA COLOMBIANA DE HISTORIA $(1942,1976)$.

\section{Referencias}

ACADEMIA COLOMBIANA DE HISTORIA. Conferencias pronunciadas por sus autores en la sala de la Academia en los años de 1940, 1941 y 1942, con ocasión de las fiestas patrias. Bogotá, D.C.: Voluntad, 1942.

. Programa de la celebración de los festejos patrios en el año de 1976. Bogotá, D.C.: Kelly, 1976.

ACEVEDO PUELLO, Rafael. La historia y la patria en la provincia de Cartagena, 1810-1814. Apuntes sobre la noción de Usos Públicos de la historia. Memoria y Sociedad, Bogotá, D.C., v. 14, n. 29, p. 7-23, 2010.

. Memorias, lecciones y representaciones históricas: la celebración del primer centenario de la Independencia en las escuelas de la provincia de Cartagena (1900-1920). Bogotá, D.C.: Universidad de los Andes, 2011.

BETANCOURT, Alexander. Pasado nacional y revisionismo histórico: lecturas sobre la independencia en los años sesenta en Colombia y México. In: RAMÍREZ, Renzo (Comp.). Estudios comparados de historia moderna y contemporânea: el caso de México y Colombia. Medellín: Universidad Nacional, 2011.

BURKE, Peter. La historia como memoria colectiva. In: Formas de historia cultural. Madrid: Alianza, 2011. p. 65-85.

CALDERÓN, María Teresa; RESTREPO, Isabela (Ed.). Colombia 1910-2010. Bogotá, D.C.: Taurus, 2010.

CHARTIER, Roger. Sociedad y escritura en la Edad Moderna: la cultura como apropiación. México, D.F.: Instituto Mora, 1995.

COLOMBIA. Ministerio del Interior y de Justicia. Ley de Víctimas y Restitución de Tierras. Por la cual se dictan medidas de atención, asistencia y reparación integral a las víctimas del conflicto armado interno y se dictan otras disposiciones. Bogotá, D.C.: Imprenta Nacional de Colombia, 2011.

Anos 90, Porto Alegre, v. 22, n. 42, p. 207-235, dez. 2015 
COLÓN, Luis Carlos. Representar la nación en el espacio urbano: Bogotá y los festejos del Centenario de la Independencia. In: GUTMAN, Margarita; MOLINOS, Rita (Ed.). Construir bicentenarios latinoamericanos en la era de la globalización. Buenos Aires: Infinito, 2012.

CONNERTON, Paul. How societies remember. New York: Cambridge University Press, 2007.

DE ALMEIDA, Jaime. Santa Librada en nuestra agenda para el Bicentenario. In: GUERRERO, Javier; WIESNER, Luis (Comp.). Memoria, historia y nación: a propósito del Bicentenario de la Independencia en Latinoamérica. Medellín: La Carreta-Universidad Pedagógica y Tecnológica de Colombia, 2010.

EARLE, Rebecca. "Padres de la Patria" and the ancestral past: commemorations of independence in nineteenth-century Spanish America. Journal of Latin American Studies, Cambridge, UK, v. 34, n. 4, p. 775-780, 2002.

EL COLECTIVO. Golpe de memoria. E-MISFÉRICA, Nueva York, n. 6.1, 2009. Disponible en: 〈http://hemisphericinstitute.org/hemi/es/el-colectivo-intro〉. Consultado en: 18 nov. 2014.

EL FANTASMA de la libertad: Celebraciones por la independencia, 1810-1910. Dirección: Jimena Montaña Cuéllar y Pablo Castillo Muñoz. Disponible en: <https://www.youtube.com/watch?v=p90GX69qDwA>. Consultado en: 20 oct. 2014.

ERLL, Astrid. Memoria colectiva y culturas del recuerdo: estudio introductorio. Bogotá, D.C.: Universidad de los Andes, 2012.

ESCOBAR, Juan Camilo. Las representaciones sociales, políticas y culturales de las independencias iberoamericanas. In: GUERRERO, Javier; WIESNER, Luis (Comp.). Memoria, historia y nación: a propósito del Bicentenario de la Independencia en Latinoamérica. Medellín: La Carreta-Universidad Pedagógica y Tecnológica de Colombia, 2010.

FISCHER, Thomas. La celebración del centenario de independencia en Bogotá y Caracas. In: SCHEUZGER, Stephan; SCHUSTER, Sven (Ed.). Los centenarios de la independencia: representaciones de la historia patria entre continuidad y cambio. Eichstätt: Zentralinstitut für Lateinamerika-Studien, 2013.

GILLIS, John (Ed.). Commemorations: the politics of national identity. Princeton: Princeton University Press, 1994.

GÓMEZ, Pablo. Patrimonio y etnopolíticas de la memoria: el pasado como aparato ideológico en la fiesta del Zocán en el templo del sol de Sogamoso. Antípoda, Bogotá, D.C., n. 12, p. 165-186, 2011.

Anos 90, Porto Alegre, v. 22, n. 42, p. 207-235, dez. 2015 
La investigación sobre las conmemoraciones rituales en Colombia...

GONZÁLEZ, Marcos. Fiesta nacional y Guerra de Independencia neogranadina. In: GUERRERO, Javier; WIESNER, Luis (Comp.). Memoria, historia y nación: a propósito del Bicentenario de la Independencia en Latinoamérica. Medellín: La Carreta-Universidad Pedagógica y Tecnológica de Colombia, 2010.

. Fiestas de independencia en Bogotá (1809-1830). In: ORTEMBERG, Pablo (Dir.). El origen de las fiestas pátrias: Hispanoamérica en la era de las Independencias. Rosario: Prohistoria, 2013.

HARTOG, François. Regímenes de historicidade: Presentismo y experiencias del tiempo. México, D.F.: Universidad Iberoamericana, 2007.

HITE, Katherine. Politics and the art of commemoration: Memorials to struggle in Latin America and Spain. London: Routledge, 2012.

INSTITUTO DISTRITAL DE CULTURA Y TURISMO (IDPC). La Ciudad de la Luz: Bogotá y la Exposición Agrícola e Industrial de 1910. Bogotá, D.C.: IDPC, 2005.

JIMÉNEZ, Andrés. Hispanismo y lengua en la conmemoración del primer Centenario de la independencia en Colombia. In: SCHEUZGER, Stephan; SCHUSTER, Sven (Ed.). Los centenarios de la independencia: representaciones de la historia patria entre continuidad y cambio. Eichstätt: Zentralinstitut für Lateinamerika-Studien, 2013.

JELIN, Elizabeth (Comp.). Las conmemoraciones: las disputas en las fechas 'in-felices'. Madrid: Siglo XXI, 2002.

JELIN, Elizabeth; LONGONI, Ana (Comp.). Escrituras, imágenes y escenarios ante la represión. Madrid: Siglo XXI, 2003.

LOMNÉ, Georges. La revolución francesa y la simbólica de los ritos bolivarianos. Historia Crítica, Bogotá, D.C., n. 5, p. 3-17, 1991.

MARTÍNEZ, Abel; OTÁLORA, Andrés. Patria y Madre Patria: las fiestas centenarias de 1910 y 1911 en Tunja. Historia y memoria, Tunja, n. 5, p. 115-143, 2012.

MARTÍNEZ, Fréderic. ¿Cómo representar a Colombia? De las exposiciones universales a la exposición del Centenario, 1851-1910. In: SÁNCHEZ, Gonzalo; WILLS, María Emma (Comp.). Museo, memoria y nación: Misión de los museos nacionales para los ciudadanos del futuro. Bogotá, D.C.: Museo Nacional de Colombia, 2000.

MEDINA, Medófilo. Bicentenario y globalización. In: GUERRERO, Javier; WIESNER, Luis (Comp.). Memoria, historia y nación: a propósito del Bicentenario de la Independencia en Latinoamérica. Medellín: La Carreta-Universidad Pedagógica y Tecnológica de Colombia, 2011.

Anos 90, Porto Alegre, v. 22, n. 42, p. 207-235, dez. 2015 
MEjÍA, Darío. ¿Qué conmemoramos? El disfraz de la diversidad entre 200 y 500 años. Maguaré, Bogotá, D.C., n. 24, p. 403-408, 2010.

MEJÍA, Germán. Noticias bibliográficas: conmemoración fiestas de la Independencia en Colombia. Memoria y Sociedad, Bogotá, D.C., v. 15, n. 30, p. 91-105, 2011.

MELO, Jorge Orlando. La celebración de la independencia: espectáculo y reflexión. 2011. Disponible en: $<$ http://www.jorgeorlandomelo.com/ambi_cele. html>. Consultado en: 20 oct. 2014.

MURILLO SANDOVAL, Juan David. Fiestas, memoria y libros: las ediciones conmemorativas del primer centenario de la Independencia en Bogotá y Cali. Memoria y Sociedad, Bogotá, D.C., v. 16, n. 33, p. 191-207, 2012.

NORA, Pierre. Pierre Nora en Les Lieux de mémoire. Montevideo: Trilce, 2008. ORTEMBERG, Pablo (Dir.). El origen de las fiestas patrias: Hispanoamérica en la era de las Independencias. Rosario: Prohistoria, 2013.

ORTEGA, Francisco; CHICANGANÁ, Yobenj (Ed.). Del dicho al hecho: 200 años de independencia y ciudadanía en Colombia, XIII Cátedra Anual de Historia Ernesto Restrepo Tirado. Bogotá, D.C.: Universidad Javeriana, 2011.

. Conceptos fundamentales de la cultura política de la Independencia. Bogotá, D.C.: Universidad Nacional; Helsinki: University of Helsinki, 2012.

PALACIOS, Marco (Ed.). Las independencias hispanoamericanas: Interpretaciones 200 años después. Bogotá, D.C.: Norma, 2009.

PÉREZ, Amada Carolina. La independencia como gesta heroica en el continuo histórico nacional: la "densidad" de la representación, 1880-1909. In: MUSEO NACIONAL DE COLOMBIA. Las historias de un grito: Doscientos años de ser colombianos - exposición conmemorativa del Bicentenario 2010. Bogotá, D.C.: Museo Nacional de Colombia, 2010.

PÉREZ, Amada Carolina; YIE, Maite. Cultura política y conmemoración en Colombia. Primer siglo de vida republicana. In: ORTEGA, Francisco; CHICANGANÁ, Yobenj (Ed.). Conceptos fundamentales de la cultura política de la Independencia. Bogotá, D.C.: Universidad Nacional; Helsinki: University of Helsinki, 2012.

PEREIRA, Alexander. Cachacos y guaches: la plebe en los festejos bogotanos del 20 de julio de 1910. Anuario Colombiano de Historia Social y de la Cultura, Bogotá, D.C., n. 38, p. 79-108, 2011.

PIEDRAHITA, Santiago Díaz. 20 de julio de 1810: referente obligado y conmemoración crítica. Boletín de antigüedades, Bogotá, D.C., v. 93, n. 833, p. 245-262, 2006. 
RAPPAPORT, Joanne. La Política de la Memoria: Interpretación Indígena de la Historia en los Andes Colombianos. Popayán: Universidad del Cauca, 2000. RESTREPO, Eduardo. ¿Quién imagina la independencia? a propósito de la celebración del bicentenario en Colombia. Nómadas, Bogotá, D.C., n. 33, p. 69-77, 2010.

RINCÓN, Carlos. Conmemoraciones y crisis: Centenario, Sesquicentenario, Bicentenario. In: ESCOBAR, Juan Camilo; DE MOJICA, Sarah; MAYA, Adolfo (Ed.). Conmemoraciones y crisis: Procesos independentistas en Iberoamérica y la Nueva Granada. Bogotá, D.C.: Universidad Javeriana-Universidad EAFIT, 2012.

ROMÁN, Raúl. Memorias enfrentadas: Centenario, nación y estado 1910-1921. Memorias: Revista Digital de Historia y Arqueología desde el Caribe, Barranquilla, n. 2, p. 1-22, 2010.

SAADE, Marta; MORA, Pablo. 200 años desde las orillas: otras voces en la conmemoración de la independencia en Colombia. Maguaré, Bogotá, D.C., n. 24, p. 397-401, 2010.

SAMACÁ, Gabriel. El centenario de la muerte del "hombre de las leyes": el papel del Centro de Historia de Santander y la conmemoración departamental (1940). Memoria y Sociedad, Bogotá, D.C., v. 17, n. 35, p. 38-57, 2013.

SCHEUZGER, Stephan. Las conmemoraciones en los Centenarios de la independencia: un comentario a su estudio historiográfico. In: SCHEUZGER, Stephan; SCHUSTER, Sven (Ed.). Los centenarios de la independencia: representaciones de la historia patria entre continuidad y cambio. Eichstätt: Zentralinstitut für Lateinamerika-Studien, 2013.

TARAZONA, Álvaro Acevedo. El primer centenario de Colombia (20 de julio de 1910): Unidad nacional, iconografías y retóricas de una conmemoración. Credencial Historia, Bogotá, D.C., n. 252, 2010.

THOMPSON, Edward Palmer. Historia y antropología. In: Agenda para una historia radical. Barcelona: Crítica, 2000. p. 13-43.

TORRES, Juan Carlos (Comp.). El gran libro del Bicentenario. Bogotá, D.C.: Planeta, 2011.

TRAVERSO, Enzo. El pasado, instrucciones de uso: Historia, memoria, política. Madrid: Marcial Pons, 2007.

VALENCIA, Feliciano. No estamos para celebraciones: las lecciones aprendidas de los 200 años. Maguaré, Bogotá, D.C., n. 24, p. 409-410, 2010. 
VANEGAS, Carolina. Representaciones de la Independencia y la construcción de una "imagen nacional" en la celebración del centenario en 1910. In: MUSEO NACIONAL DE COLOMBIA. Las historias de un grito: Doscientos años de ser colombianos - exposición conmemorativa del Bicentenario 2010. Bogotá, D.C.: Museo Nacional de Colombia, 2010.

. Disputas simbólicas en la celebración del centenario de la independencia de Colombia en Bogotá (1910): los monumentos a Simón Bolívar y a Policarpa Salavarrieta. Bogotá, D.C.: Ministerio de Cultura, 2012.

VARGAS, Sebastián. El bicentenario de la independencia en Colombia: rituales, documentos, reflexiones. Memoria y Sociedad, Bogotá, D.C., v. 15, n. 31, p. 66-84, 2011.

Presentación del dossier: políticas de la memoria y usos públicos de la historia. Memoria y Sociedad, Bogotá, D.C., v. 17, n. 35, p. 6-9, 2013.

. Cabalgando entre el pasado y el presente: Usos estatales del Bicentenario de la Independencia en Colombia (2009-2010). In: GUTMAN, Margarita; Versace, Ileana (Ed.). Bicentenarios en Acción: Conmemoración, Crisis Económica y Movilización Política en América Latina. Buenos Aires: Infinito. En prensa.

YIE, Maite. Narrativas de pasado, de nación y ciudadanía en las celebraciones patrióticas durante el siglo XX en Colombia. In: MUSEO NACIONAL DE COLOMBIA. Las historias de un grito: Doscientos años de ser colombianos exposición conmemorativa del Bicentenario 2010. Bogotá, D.C.: Museo Nacional de Colombia, 2010.

ZAMBRANO, Fabio; CASTELBLANCO, Carolina. El kiosco de la luz y el discurso de la modernidad. Bogotá, D.C.: Alcaldía Mayor, 2002.

Recebido em: 03/12/2014

Aprovado em: 01/02/2015

Anos 90, Porto Alegre, v. 22, n. 42, p. 207-235, dez. 2015 